\title{
IMPACTO DE LA IMPLEMENTACIÓN DEL PLAN AUGE EN LA DETECCIÓN ANTENATAL DE CARDIOPATÍAS CONGÉNITAS
}

\author{
Felipe Concha D. ${ }^{a}$, Nicole Pastén V. ${ }^{a}$, Victoria Espinosa F. PhD ${ }^{1 b}$, Fanny \\ López A. PhD $2 a$ \\ ${ }^{1}$ Escuela de Medicina, Laboratorio de Investigación Científica Emory Black, Facultad de Ciencias Médicas, Univer- \\ sidad de Santiago de Chile. ${ }^{2}$ Escuela de Obstetricia y Puericultura, Facultad de Ciencias Médicas, Universidad de \\ Santiago de Chile.
}

a Matrón/a. ${ }^{\mathrm{b}}$ Bioquímica.

\section{RESUMEN}

Antecedentes: El plan AUGE recomienda para detectar cardiopatías congénitas (CC), la realización de ecografía obstétrica de pesquisa y ecocardiografía fetal confirmatoria. Estos elementos son indispensables para informar adecuadamente a las familias y organizar en forma óptima el cuidado perinatal, estableciendo continuidad con el manejo postnatal y mejorar la oportunidad de vida del recién nacido. Objetivo: Determinar el impacto del plan AUGE en la frecuencia de detección perinatal de cardiopatías congénitas, a través de un estudio comparativo de la incidencia de estas patologías detectadas en el periodo 2000-2002 (pre AUGE) y 2003-2005 (AUGE), registradas en la Unidad de Neonatología del Hospital San José, evaluando la resolución de los casos en el Hospital Roberto del Río. Método: Estudio de tipo observacional, descriptivo, retrospectivo, de corte longitudinal, con una muestra no probabilística, recogida por acumulación de fichas clínicas del Hospital San José en la detección antenatal, y los datos de resolución quirúrgica del Hospital Roberto del Río de 231 recién nacidos. Resultados: Se encontraron diferencias estadísticamente significativas $(p<0,0001)$, en la distribución por tipo de CC detectadas ante y postnatalmente en el periodo AUGE y en la distribución por tipo de CC detectadas antenatalmente entre los periodos pre-AUGE y AUGE $(p=0,0467)$. Conclusiones: La implementación del plan AUGE, produjo un mayor número de diagnósticos de CC, todo esto determinó una mayor sobrevida neonatal.

\section{PALABRAS CLAVE: Cardiopatía congénita, plan AUGE, diagnóstico prenatal}

\section{SUMMARY}

Background: The AUGE plan (spanish initials for Universal Access with Explicit Guarantees in Health), is the realization of a screening through obstetric ultrasonography to detect congenital hearth disease (CHD) and fetal echocardiography to confirm diagnosis. These elements are indispensable to be able to inform appropriately to the families and to organize in good form the prenatal care, establishing continuity with the postnatal handling and to improve the opportunity of life of the newly born. Objective: To determine the impact of the plan peak in the frequency of prenatal detection of $\mathrm{CHD}$, through a comparative study of the incidence of these pathologies detected in the period 2000-2002 (pre AUGE) and 2003-2005 (AUGE), registered in the Neonatology Unit of the Hospital San José, evaluating the resolution of the clinical cases in the Hospital Roberto del Río. Method: Descriptive study, non experimental, retrospective of longitudinal court, 
with a non probabilistic sample, picked up by accumulation of clinical records of the Hospital San José (prenatal detection) and the data of surgical resolution of the Hospital Roberto del Río of 231 newborns. Results: There were significant statistically differences $(p<0.0001)$ as for the incidence of the prenatal and postnatal detection of CHD in AUGE and in the prenatal detection of congenital hearth diseases, among the periods pre AUGE and AUGE ( $p=0.0467)$. Conclusions: Implementing the AUGE, produced a greater number of CHD diagnoses, all this produced a higher neonatal survival.

\section{KEY WORDS: Congenital heart disease, AUGE plan, prenatal detection}

\section{INTRODUCCIÓN}

La formulación de los objetivos sanitarios elaborados por el Ministerio de Salud de Chile para la década $2000-2010$, tiene como punto inicial en el año 2000 , una tasa de mortalidad infantil de 8,9 por 1.000 nacidos vivos, la que pretende disminuir a 7,5 por 1.000 nacidos vivos para el año $2010(1,2)$. La tasa de mortalidad infantil, ha sido tradicionalmente reconocida como uno de los indicadores más sensibles de la situación de salud y del nivel de vida de una comunidad (3).

En Chile, a partir de la década de los años 80, comienza a adquirir importancia como causas de muerte, las afecciones del período perinatal, especialmente asociadas al bajo peso al nacer y las malformaciones congénitas (4). Estas últimas, originaron un $23 \%$ de las muertes en menores de 1 año en 1990 y un $34 \%$ en el año 2000, ocupando el segundo lugar como causa de muerte en menores de 1 año (5). Así, para alcanzar los índices propuestos en los objetivos sanitarios, se debe reducir uno de sus componentes más relevantes, como son las cardiopatías congénitas, porque a medida que los países mejoran sus índices de salud, las malformaciones congénitas pasan a ocupar uno de los primeros lugares en las causas de mortalidad infantil (4-7).

Con el propósito de cumplir las metas y teniendo presente que en nuestro país actualmente, la reducción de la mortalidad infantil ha estado más ligada a las intervenciones de salud, que al mejoramiento de las condiciones socioeconómicas (4), se establecieron 49 objetivos de impacto, distribuidos en áreas de salud infantil, salud de la mujer y enfermedades infecciosas que utilizan como instrumentos las políticas de salud pública, principalmente el programa de salud de la mujer (8) que ha demostrado desde su creación, un gran impacto en el descenso de los índices de morbi-mortalidad materno infantil (9).

Para continuar con tendencia, han sido publicadas la Guía Perinatal en 2003 (10) y la Guía de Car- diopatías Congénitas en 2005 (11), que permiten dirigir, uniformizar y evaluar los procesos técnicos, todo esto inmerso en el marco de la actual Reforma de Salud Chilena, el Plan de Acceso Universal con Garantías Explícitas - AUGE, que tiene como objetivo garantizar la equidad en el acceso de la población a la salud, independientemente de la capacidad de pago de las personas (12) con lo anterior se pretende contribuir ha alcanzar los objetivos sanitarios en el año 2010.

El objetivo de este estudio es comparar la frecuencia de detección de las cardiopatías congénitas en el periodo perinatal de los partos atendidos en el Hospital San José, en el periodo 2000-2002 (pre-AUGE) con el periodo 2003-2005 (AUGE).

\section{MATERIAL Y MÉTODO}

Estudio de diseño observacional, descriptivo, comparativo, retrospectivo de corte longitudinal. Corresponde a las datos de 52.477 partos que se atendieron en el Hospital San José entre los años 2000 a 2005, de los cuales fueron seleccionados 231 casos que presentaron una cardiopatía congénita e ingresaron a la Unidad de Neonatología del Hospital San José. En los casos que la cardiopatía congénita tuvo resolución quirúrgica fueron recolectados los datos de las fichas clínicas del Hospital de Niños Dr. Roberto del Río. Las variables analizadas fueron: detección prenatal de cardiopatía congénita (CC), detección postnatal de CC, factores de riesgo maternos y fetales para desarrollar una $\mathrm{CC}$, tipo de $\mathrm{CC}$, método de detección prenatal de $\mathrm{CC}$, método de detección postnatal de $\mathrm{CC}$ y tipo de resolución de caso con $\mathrm{CC}$ en los periodos definidos como pre AUGE (2000-2002) y AUGE (2003-2005).

Se utilizaron en el análisis la determinación de medidas de tendencia central de las frecuencias para cada variable. El análisis estadístico inferencial se realizó a través de las pruebas $U$ de Mann-Whitney con un nivel de significación del 5\% $(p \leq 0,05)$, utilizando el programa estadístico Graphpad Prism 4.02 


\section{RESULTADOS}

La incidencia de cardiopatías congénitas detectadas entre los partos atendidos en el Hospital San José, en los 3 años previos al plan AUGE, tuvo un promedio de $0,4 \%$, mientras que en los 3 años iniciales del periodo AUGE alcanzó un promedio de $0,5 \%$ (Tabla I).

En cuanto a los periodos de detección de las $\mathrm{CC}$, hubo un mayor número de casos detectados en la fase postnatal, respecto de los casos detectados en el antenatal, en ambos periodos de estudio, sin que esta diferencia fuese estadísticamente significativa (Tabla II). Al observar la totalidad de la población, se encontró un mayor número de casos detectados en el periodo AUGE, aun cuando hubo un mayor número de partos en el periodo pre AUGE (Tabla II).

De las cardiopatías congénitas detectadas, destacan por su gran diferencia de frecuencia de detección, entre los periodos pre y postnatal respecto de las demás patologías, la comunicación interauricular (CIA), la comunicación interventricular (CIV) y el ductus arterioso persistente (DAP) (Tablas Illa y b). Del análisis estadístico de esta información se obtuvo que existe diferencia estadísticamente significativa $(p<0,0001)$ en la distribución de CC detectadas ante y postnatalmente en los periodos pre AUGE y AUGE. Se determinó además que existe diferencia estadísticamente significativa $(p=0,0467)$ en la distribución de $C C$ detectadas antenatalmente en el periodo pre-AUGE y AUGE y que no existe diferencia estadísticamente significativa $(p=0,4137)$ en la distribución de CC detectadas postnatalmente en el periodo pre-AUGE y AUGE (Tablas Illa y b).

Se observó que un tercio de los recién nacidos con CC (37\% en periodo pre AUGE y $33 \%$ en AUGE), presentaban algún factor de riesgo materno y/o fetal. En los criterios de riesgo materno, se destaca principalmente la edad materna menor a 18 y mayor de 35 años, tanto en el periodo pre AUGE como AUGE. Diferente es el caso del criterio de riesgo fetal, en la cual aparecen varios de ellos con mayor frecuencia en ambos periodos pre AUGE y AUGE. El análisis estadístico indica que no existe diferencia estadísticamente significativa en la distribución de los criterios de riesgo maternos ni fetales asociadas a CC detectadas, entre los periodos pre AUGE y AUGE $(p=0,1049$ y $p=0,5737$, respectivamente) (Tabla IV).

Tabla I

INCIDENCIA DE CARDIOPATÍAS CONGÉNITAS DETECTADAS ENTRE LOS PARTOS ATENDIDOS EN EL HOSPITAL SAN JOSÉ, EN LOS PERIODOS PRE AUGE (2000-2002) Y AUGE (2003-2005)

\begin{tabular}{|c|c|c|c|c|c|c|c|}
\hline \multirow[b]{2}{*}{ Periodo } & \multirow[b]{2}{*}{ año } & \multicolumn{2}{|c|}{$\begin{array}{l}\text { Sin cardiopatía } \\
\text { congénita }\end{array}$} & \multicolumn{2}{|c|}{$\begin{array}{c}\text { Con cardiopatía } \\
\text { congénita }\end{array}$} & \multicolumn{2}{|c|}{ Total } \\
\hline & & $\mathrm{n}$ & $\%$ & $\mathrm{n}$ & $\%$ & $\mathrm{n}$ & $\%$ \\
\hline Pre & 2000 & 8869 & 99,7 & 27 & 0,3 & 8896 & 33,1 \\
\hline \multirow[t]{4}{*}{ AUGE } & 2001 & 9041 & 99,6 & 35 & 0,4 & 9076 & 33,8 \\
\hline & 2002 & 8852 & 99,6 & 35 & 0,4 & 8887 & 33,1 \\
\hline & Total & 26762 & 99,6 & 97 & 0,4 & 26859 & 100 \\
\hline & año & $\mathrm{n}$ & $\%$ & $\mathrm{n}$ & $\%$ & $n$ & $\%$ \\
\hline \multirow[t]{4}{*}{ AUGE } & 2003 & 8694 & 99,5 & 45 & 0,5 & 8739 & 33,8 \\
\hline & 2004 & 8421 & 99,5 & 36 & 0,5 & 8457 & 32,7 \\
\hline & 2005 & 8600 & 99,4 & 53 & 0,6 & 8653 & 33,5 \\
\hline & Total & 25715 & 99,5 & 134 & 0,5 & 25849 & 100 \\
\hline
\end{tabular}

Por test $U$ de Mann Whitney no existe diferencia estadísticamente significativa en la distribución de partos sin CC del periodo pre AUGE y AUGE $(p=0,1)$. De igual manera no existe diferencia en el número de partos con $C C$ atendidos en el periodo pre AUGE y AUGE $(p=0,1)$ 
Tabla II

DISTRIBUCIÓN DE LOS RECIÉN NACIDOS INGRESADOS A LA UNIDAD DE NEONATOLOGÍA DEL HOSPITAL SAN JOSÉ EN LOS PERIODOS PRE AUGE Y AUGE, SEGÚN PERIODOS DE DETECCIÓN DE LAS CARDIOPATÍAS CONGÉNITAS

\begin{tabular}{lcccccc}
\hline \multirow{2}{*}{ Periodo } & \multicolumn{2}{c}{ Detección prenatal } & \multicolumn{2}{c}{ Detección postnatal } & Total \\
& Año & $\mathrm{n}$ & $\%$ & $\mathrm{n}$ & $\%$ & $\mathrm{n}$ \\
\hline \multirow{2}{*}{ Pre } & 2000 & 1 & 0,04 & 26 & 0,96 & 27 \\
\multirow{4}{*}{ AUGE } & 2001 & 3 & 0,09 & 32 & 0,91 & 35 \\
& 2002 & 4 & 0,11 & 31 & 0,89 & 35 \\
& Total & 8 & 0,08 & 89 & 0,92 & 97 \\
& & & & & & 45 \\
& 2003 & 9 & 0,20 & 36 & 0,80 & 36 \\
& 2004 & 8 & 0,22 & 28 & 0,78 & 53 \\
& 2005 & 12 & 0,23 & 41 & 0,77 & 134 \\
\hline
\end{tabular}

No existen diferencias estadísticamente significativas entre el numero de casos detectados postnatal con respecto a los prenatal para ambos periodos ( $p=0,1$ en ambos casos). No existe diferencia estadísticamente significativa en el numero de casos con detección prenatal y postnatal al comparar entre los periodos pre AUGE y AUGE ( $p=0,1$ y 0,4 respectivamente)

Respecto de los métodos de detección de CC, se observó que en el periodo prenatal, con la implementación del plan AUGE, se introdujo la utilización de ecocardiografia fetal resultando que para el diagnostico CC había un método de sospecha y otro confirmatorio, por el contrario en la detección postnatal se utilizó solo un método de detección que correspondió a ecocardiografía en la mayoría de los casos tanto en el periodo pre AUGE como AUGE (Tabla V).

Por último al analizar la resolución de la CC con detección antenatal más frecuente durante el periodo pre AUGE, se observó que fue el fallecimiento, mientras que en el periodo AUGE fue la resolución quirúrgica, en los casos con detección postnatal, fue la interconsulta, sin que haya diferencia estadísticamente significativa $(p \leq 0,05)$ entre los grupos con detección ante y post natal en los periodos pre AUGE y AUGE (Tabla VI).

\section{DISCUSIÓN}

La Red Asistencial del Servicio de Salud Metropolitano Norte (SSMN), en Santiago de Chile, tiene como Hospital Base al Hospital San José, que atiende la mayoría de los partos de esta región y al Hospital de Niños Dr. Roberto del Río como Centro de Referencia Nacional Infantil de Cardiología y Cardiocirugía.
Los resultados obtenidos a partir de la población de estudio, evidencian una incidencia de cardiopatías congénitas en los 3 años previos al plan AUGE de 3,62 /1.000 nacidos vivos y en los 3 años iniciales de plan AUGE de 5,21/1.000 recién nacidos.

En Cuba, país que posee un sistema organizado y gratuito de acceso a la salud, en la provincia de Villa Clara se realizó un estudio descriptivo entre en los años 2000-2002, coincidente con el periodo pre AUGE, obteniéndose una incidencia de 10,77/1.000 nacidos vivos, según sus autores, este índice se debe a la fortaleza de la red cardiopediátrica (13).

En EEUU de Norte América, región con mayores recursos económicos y con un sistema de salud organizado, la incidencia de CC en la década de los 90's paso de 3 a 12/1000 nacidos vivos debido a un mejoramiento en la capacitación de cardiólogos pediátricos, un diagnósticos precoz y correcto y el advenimiento de la ecocardiografía Doppler color (14). Esta incidencia es aproximada a la estimada en la población mundial de 8 a 12/1000 nacidos vivos (15). Al comparar estos índices con los de nuestro estudio, verificamos que la implantación del plan AUGE ha mejorado la cobertura y detección de CC aproximándose al cumplimiento de los objetivos sanitarios elaborados por el Ministerio de Salud de Chile para la década $2000-2010$.

Respecto de la incidencia de CC en relación al 
Tabla III a

DISTRIBUCIÓN DEL TIPO DE CARDIOPATÍAS CONGÉNITAS DETECTADAS EN PERIODO
PRENATAL Y POSTNATAL ENTRE LOS RECIÉN NACIDOS INGRESADOS A LA UNIDAD DE
NEONATOLOGÍA DEL HOSPITAL SAN JOSÉ, 2000-2002. PRE AUGE

\begin{tabular}{|c|c|c|c|c|c|c|}
\hline \multirow[t]{2}{*}{ Patologías } & \multicolumn{2}{|c|}{ Detección antenatal } & \multicolumn{2}{|c|}{ Detección postnatal } & \multicolumn{2}{|c|}{ Total } \\
\hline & $\mathrm{n}$ & $\%$ & $\mathrm{n}$ & $\%$ & $\mathrm{n}$ & $\%$ \\
\hline Comunicación IV & 1 & 0,2 & 41 & 0,21 & 42 & 0,21 \\
\hline Comunicación IA & 1 & 0,2 & 42 & 0,22 & 43 & 0,21 \\
\hline Hipoplasia VD & 1 & 0,2 & 3 & 0,01 & 4 & 0,01 \\
\hline Hipoplasia VI & 0 & 0 & 1 & 0,01 & 1 & 0,01 \\
\hline Tetralogía de Fallot & 0 & 0 & 5 & 0,02 & 5 & 0,02 \\
\hline Ventrículo único & 0 & 0 & 4 & 0,02 & 4 & 0,01 \\
\hline Doble arco aórtico & 0 & 0 & 1 & 0,01 & 1 & 0,01 \\
\hline TGA/V & 0 & 0 & 7 & 0,03 & 7 & 0,04 \\
\hline Atresia pulmonar & 0 & 0 & 1 & 0,01 & 1 & 0,01 \\
\hline Atresia tricuspidea & 0 & 0 & 5 & 0,02 & 5 & 0,02 \\
\hline Atresia mitral & 0 & 0 & 2 & 0,01 & 2 & 0,01 \\
\hline Atresia aórtica & 0 & 0 & 2 & 0,01 & 2 & 0,01 \\
\hline Aurícula única & 0 & 0 & 1 & 0,01 & 1 & 0,01 \\
\hline Coartación aórtica & 0 & 0 & 5 & 0,02 & 5 & 0,02 \\
\hline Canal AV completo & 0 & 0 & 4 & 0,02 & 4 & 0,01 \\
\hline Válvula AV única & 0 & 0 & 2 & 0,01 & 2 & 0,01 \\
\hline Aplasia válvulas pulmonares & 0 & 0 & 1 & 0,01 & 1 & 0,01 \\
\hline Drenaje venoso anómalo & 0 & 0 & 2 & 0,01 & 2 & 0,01 \\
\hline DAP & 1 & 0,2 & 43 & 0,22 & 44 & 0,23 \\
\hline FOP & 0 & 0 & 14 & 0,08 & 14 & 0,07 \\
\hline ERP & 0 & 0 & 7 & 0,03 & 7 & 0,04 \\
\hline Tronco arterioso tipo IV & 1 & 0,2 & 1 & 0,01 & 2 & 0,01 \\
\hline No especificado (CC compleja) & 0 & 0 & 3 & 0,01 & 3 & 0,01 \\
\hline Total & 5 & 1,0 & 197 & 1,00 & 202 & 1,00 \\
\hline
\end{tabular}

IV: interventricular. IA: interauricular. VD: ventrículo derecho. VI: ventrículo izquierdo. TGA/V: transposición de grandes arterias/vasos; DAP: ductus arterioso persistente; FOP: foramen oval permeable; ERP: Estenosis de rama pulmonar. Existe diferencia estadísticamente significativa $(p<0,0001)$ en la distribución de cardiopatías congénitas detectadas ante y postnatalmente en el periodo pre-AUGE.

periodo de su detección, observamos en nuestra población, que durante el periodo AUGE, la pesquisa de CC en la fase prenatal fue de 1,12/1.000 nacidos vivos, mayor que la del periodo pre AUGE con $0,29 / 1.000$ nacidos vivos. En la literatura científica no existe consenso en el beneficio de la precocidad de este diagnostico $(5,16)$, ya que ha sido difícil demostrar la utilidad real de la detección prenatal de las cardiopatías, porque la mayoría de los informes corresponden a partos atendidos en centros de alta complejidad con facilidades para derivar, lo cual eventualmente evita la demora en el manejo de los neonatos sin diagnóstico prenatal. Esto último es la situación chilena, que en el periodo previo a la implantación del plan AUGE, un 33\% de los niños que fallecían por cardiopatía congénita durante el primer año de vida, lo hacían en "espera de diagnóstico cardiológico", definida como el lapso entre la sospecha clínica y el estudio cardiológico (11). Por lo tanto, en Chile que por sus características geopolíticas que concentra los centros de alta complejidad en la Región Metropolitana, hace que el diagnostico precoz sea una medida efectiva, la cual es preconizada en la Guía Clínica de CC (2005) con plazos definidos de tratamiento.

Lo que esta evidenciado en la literatura, es el beneficio del diagnóstico prenatal para patologías congénitas no cianóticas, con cortocircuito de iz- 
Tabla III b

\section{DISTRIBUCIÓN DEL TIPO DE CARDIOPATÍAS CONGÉNITAS DETECTADAS EN PERIODO PRENATAL Y POSTNATAL ENTRE LOS RECIÉN NACIDOS INGRESADOS A LA UNIDAD DE NEONATOLOGÍA DEL HOSPITAL SAN JOSÉ, 2003-2005. AUGE}

\begin{tabular}{|c|c|c|c|c|c|c|}
\hline \multirow[t]{2}{*}{ Patologías } & \multicolumn{2}{|c|}{ Detección antenatal } & \multicolumn{2}{|c|}{ Detección postnatal } & \multicolumn{2}{|c|}{ Total } \\
\hline & $\mathrm{n}$ & $\%$ & $\mathrm{n}$ & $\%$ & $\mathrm{n}$ & $\%$ \\
\hline Comunicación IV & 3 & 0,14 & 69 & 0,28 & 72 & 0,27 \\
\hline Comunicación IA & 3 & 0,14 & 37 & 0,15 & 40 & 0,15 \\
\hline Hipoplasia VD & 1 & 0,05 & 6 & 0,02 & 7 & 0,03 \\
\hline Hipoplasia VI & 4 & 0,19 & 5 & 0,02 & 9 & 0,03 \\
\hline Tetralogía de Fallot & 1 & 0,05 & 10 & 0,04 & 11 & 0,04 \\
\hline Ventrículo único & 1 & 0,05 & 2 & 0,01 & 3 & 0,01 \\
\hline Enfermedad de Ebstein & 1 & 0,05 & 4 & 0,02 & 5 & 0,02 \\
\hline TG A/V & 0 & 0,00 & 4 & 0,02 & 4 & 0,02 \\
\hline Atresia pulmonar & 0 & 0,00 & 4 & 0,02 & 4 & 0,02 \\
\hline Atresia tricuspidea & 1 & 0,05 & 6 & 0,02 & 7 & 0,03 \\
\hline Atre sia mitral & 2 & 0,10 & 3 & 0,01 & 5 & 0,02 \\
\hline Atresia aórtica & 0 & 0,00 & 1 & 0,00 & 1 & 0,00 \\
\hline Aurícula única & 1 & 0,05 & 1 & 0,00 & 2 & 0,01 \\
\hline Coartación aórtica & 0 & 0,00 & 2 & 0,01 & 2 & 0,01 \\
\hline Canal AV completo & 0 & 0,00 & 9 & 0,04 & 9 & 0,03 \\
\hline DSVD & 1 & 0,05 & 0 & 0,00 & 1 & 0,00 \\
\hline Válvula AV ún ica & 0 & 0,00 & 3 & 0,01 & 3 & 0,01 \\
\hline Aplasia válvulas pulmo nares & 1 & 0,05 & 0 & 0,00 & 1 & 0,00 \\
\hline Pentalogía de Fallot & 0 & 0,00 & 1 & 0,00 & 1 & 0,00 \\
\hline Corazón 5 cavidades & 0 & 0,00 & 1 & 0,00 & 1 & 0,00 \\
\hline Estenosis mitral & 0 & 0,00 & 3 & 0,01 & 3 & 0,01 \\
\hline Estenosis válvula aórtica & 0 & 0,00 & 3 & 0,01 & 3 & 0,01 \\
\hline Drenaje venoso anómalo & 0 & 0,00 & 2 & 0,01 & 2 & 0,01 \\
\hline DAP & 1 & 0,05 & 40 & 0,16 & 41 & 0,15 \\
\hline FOP & 0 & 0,00 & 13 & 0,05 & 13 & 0,05 \\
\hline ERP & 0 & 0,00 & 15 & 0,06 & 15 & 0,06 \\
\hline No especificado (CC compleja) & 0 & 0,00 & 1 & 0,00 & 1 & 0,00 \\
\hline Total & 21 & 1,00 & 245 & 1,00 & 266 & 1,00 \\
\hline
\end{tabular}

IV: interventricular. IA: interauricular. VD: ventrículo derecho. VI: ventrículo izquierdo. TGA/V: transposición de grandes arterias/vasos; DAP: ductus arterioso persistente; FOP: foramen oval permeable; ERP: Estenosis de rama pulmonar; DSVD: doble salida del ventrículo derecho. Según U de Mann-Whitney: Existe diferencia estadísticamente significativa $(p<0,0001)$ en la distribución de cardiopatías congénitas (CC) detectadas ante y postnatalmente en el periodo AUGE; existe diferencia estadísticamente significativa $(p=0,0467)$ en la distribución de CC detectadas antenatalmente en el periodo pre-AUGE y AUGE; no existe diferencia estadísticamente significativa $(p=0,4137)$ en la distribución de $C C$ detectadas postnatalmente en el periodo pre-AUGE y AUGE.

quierda a derecha, que aparecen diagnosticadas con mayor frecuencia en nuestra población de estudio (16), resultados similares a los detectados en un estudio realizado en el Hospital Italiano de Buenos Aires, Argentina, en el que se cuantificaron los tipos de cardiopatías congénitas diagnosticadas entre $1998-2000$ (17).
La tendencia actual se orienta principalmente a la detección de patologías, que a la evaluación del grado de riesgo, porque los "factores de riesgo" no parecen ser un buen predictor de aquellos embarazos que presentarán complicaciones, ya que, la mayoría de las mujeres que sufren complicaciones son consideradas de "bajo riesgo" y a la inversa, la 
Tabla IV

DISTRIBUCIÓN DE LOS CRITERIOS DE RIESGOS MATERNOS Y FETALES ASOCIADOS A CARDIOPATÍAS CONGÉNITAS DETECTADAS EN LOS RECIÉN NACIDOS INGRESADOS A LA UNIDAD DE NEONATOLOGÍA DEL HOSPITAL SAN JOSÉ EN LOS PERIODOS PRE AUGE Y AUGE

\begin{tabular}{|c|c|c|c|c|c|}
\hline \multirow[b]{2}{*}{ Criterios de riesgo maternos } & \multicolumn{2}{|c|}{ Pre AUGE } & \multicolumn{2}{|c|}{ AUGE } & \multirow[t]{2}{*}{ Total } \\
\hline & $\mathrm{n}$ & $\%$ & $\mathrm{n}$ & $\%$ & \\
\hline Edad materna $(<18 \mathrm{y}>35 \mathrm{a})$ & 44 & 0,81 & 24 & 0,44 & 68 \\
\hline Embarazo múltiple & 0 & 0 & 5 & 0,10 & 5 \\
\hline Exposición a teratógenos & 0 & 0 & 4 & 0,07 & 4 \\
\hline Infección viral específica & 3 & 0,06 & 4 & 0,07 & 7 \\
\hline Epilepsia & 0 & 0 & 1 & 0,02 & 1 \\
\hline Lupus eritematoso sistémico & 1 & 0,02 & 3 & 0,06 & 4 \\
\hline Incompatibilidad Rh & 4 & 0,07 & 2 & 0,04 & 6 \\
\hline Diabetes g/preg & 2 & 0,04 & 11 & 0,20 & 13 \\
\hline \multirow[t]{2}{*}{ Total } & 54 & 1,00 & 54 & 1,00 & 108 \\
\hline & \multicolumn{2}{|c|}{ Pre AUGE } & \multicolumn{2}{|c|}{ AUGE } & Total \\
\hline Criterios de riesgo fetales & $n$ & $\%$ & $\mathrm{n}$ & $\%$ & \\
\hline Malformación congénita & 23 & 0,28 & 20 & 0,16 & 43 \\
\hline Aberración cromosómica & 18 & 0,21 & 35 & 0,27 & 53 \\
\hline Genopatía & 3 & 0,04 & 6 & 0,05 & 9 \\
\hline Antecedente familiar de CC o & 2 & 0,02 & 2 & 0,02 & 4 \\
\hline \multicolumn{6}{|l|}{ hijo previo con CC } \\
\hline Crecimiento fetal anormal & 18 & 0,21 & 31 & 0,24 & 49 \\
\hline Oligoamnios- polihidroamnios & 7 & 0,08 & 16 & 0,12 & 23 \\
\hline Hidrops fetal & 2 & 0,02 & 2 & 0,02 & 4 \\
\hline F/R cardiaco anormal & 12 & 0,14 & 16 & 0,12 & 28 \\
\hline Total & 85 & 1,00 & 128 & 1,00 & 213 \\
\hline
\end{tabular}

g/preg: gestacional/pregestacional. F/R: frecuencia o ritmo. No existe diferencia estadísticamente significativa en la distribución de los criterios de riesgo maternos ni fetales asociadas a cardiopatías congénitas detectadas, entre los periodos pre AUGE y AUGE ( $p=0,1049$ y $p=0,5737$, respectivamente) 
Tabla V

DISTRIBUCIÓN DE LOS MÉTODOS DE DETECCIÓN DE CARDIOPATÍAS CONGÉNITAS UTILIZADOS EN LOS RECIÉN NACIDOS INGRESADOS A LA UNIDAD DE NEONATOLOGÍA DEL HOSPITAL SAN JOSÉ EN LOS PERIODOS PRE AUGE Y AUGE

\begin{tabular}{|c|c|c|c|c|c|}
\hline \multirow[b]{2}{*}{ Detección prenatal } & \multicolumn{2}{|c|}{ Pre AUGE } & \multicolumn{2}{|c|}{ AUGE } & \multirow[b]{2}{*}{ Tota } \\
\hline & $\mathrm{n}$ & $\%$ & $\mathrm{n}$ & $\%$ & \\
\hline EOR & 3 & 1,00 & 2 & 0,25 & 5 \\
\hline ECGF & 0 & 0 & 6 & 0,75 & 6 \\
\hline \multirow[t]{2}{*}{ Total } & 3 & 1,00 & 8 & 1,00 & 11 \\
\hline & \multicolumn{2}{|c|}{ Pre AUGE } & \multicolumn{2}{|c|}{ AUGE } & \\
\hline Detección postnatal & $\mathrm{n}$ & $\%$ & $n$ & $\%$ & Total \\
\hline ECG & 91 & 0,99 & 114 & 1,00 & 205 \\
\hline ECO & 1 & 0,01 & 0 & 0 & 1 \\
\hline Total & 92 & 1,00 & 114 & 1,00 & 206 \\
\hline
\end{tabular}

EOR: ecografia obstetrica de rutina. ECGF: ecocardiografia fetal. ECO: ecocardiograma. No existe diferencia estadísticamente significativa entre los métodos de detección prenatal pre AUGE y AUGE $(p=0,4226)$. No hay diferencia estadísticamente significativa, entre los métodos de detección post natal en el periodo pre AUGE y AUGE $(p=0,8935)$.

gran mayoría de las mujeres consideradas de "alto riesgo" cursan sus embarazos sin presentar mayores problemas (8). Esto confirmado por trabajos que han descrito la ausencia de factores de riesgo materno hasta en un $92 \%$ de los fetos con CC y apenas un $10 \%$ de las CC se presentan en embarazadas con factores de riesgo $(18,19)$. Concordando con lo antes descrito, en nuestro estudio se detectaron recién nacidos con $\mathrm{CC}$ tanto en el periodo pre AUGE como en el AUGE, proveniente de madres sin factores de riesgo para CC (63\% y $67 \%)$.

Teniendo presente que la mayoría de las CC ocurre en gestaciones sin factores de riesgo, adquiere mayor valor los métodos de detección como la ecografía obstétrica de rutina (EOR) que está incorporada en el programa de control prenatal. En nuestra población de estudio, la EOR aparece como método de diagnostico único y preferencial para CC en el periodo pre AUGE, ya en el AUGE aparece la ecocardiografía fetal confirmatoria y la relación recién nacido: método de diagnóstico es de 1:1,5. De las 3 EOR preconizados en el programa de control prenatal, la de mayor utilidad es la realizada entre las 18 y 24 semanas de gestación que representa una oportunidad para el diagnostico de anomalías congénitas estructurales y su derivación a centros terciarios. La EOR y la ecocardiografía fetal son técnicas de diagnóstico que aparecen en nuestro estudio en el periodo AUGE y que han sido reafirmadas en la normativa de intervención del plan AUGE, basándose en que el diagnóstico prenatal de las cardiopatías congénitas permitiría reducir la mortalidad perinatal $(10,16,20)$.

Previo a la implementación del piloto Acceso Universal para prestaciones integrales y Garantías Explícitas asociadas a la atención de prioridades (AUGE), en 2002, un 33\% de los niños que fallecían por cardiopatía congénita durante el primer año de vida, lo hacían en "espera de diagnóstico cardiológico", definida como el lapso entre la sospecha clínica y el estudio cardiológico. El diagnóstico y cirugía precoz de estas malformaciones evita el daño secundario y progresivo de órganos como el corazón, pulmones y sistema nervioso central principalmente, transformándolas en malformaciones de buen pronóstico (11). El tipo de resolución para las cardiopatía congénita durante el periodo pre AUGE, resultó ser mayormente la interconsulta. 
Tabla VI

DISTRIBUCIÓN DEL TIPO DE RESOLUCIÓN UTILIZADA PARA CARDIOPATÍAS CONGÉNITAS EN LOS RECIÉN NACIDOS INGRESADOS A LA UNIDAD DE NEONATOLOGÍA DEL HOSPITAL SAN JOSÉ EN LOS PERIODOS PRE AUGE Y AUGE

\begin{tabular}{|c|c|c|c|c|c|}
\hline \multirow{2}{*}{$\begin{array}{l}\text { Tipo de resolución } \\
\text { de CC detectada } \\
\text { antenatal }\end{array}$} & \multicolumn{2}{|c|}{ Pre AUGE } & \multicolumn{2}{|c|}{ AUGE } & \multirow[t]{2}{*}{ Total } \\
\hline & $\mathrm{n}$ & $\%$ & $\mathrm{n}$ & $\%$ & \\
\hline Alta & 0 & 0 & 1 & 0,03 & 1 \\
\hline Interconsulta & 2 & 0,25 & 10 & 0,35 & 12 \\
\hline Quirúrgica & 2 & 0,25 & 11 & 0,38 & 13 \\
\hline Fallece & 4 & 0,50 & 7 & 0,24 & 11 \\
\hline Total & 8 & 1,00 & 29 & 1,00 & 37 \\
\hline Tipo de resolución & \multicolumn{2}{|c|}{ Pre AUGE } & \multicolumn{2}{|c|}{ AUGE } & Total \\
\hline post natal & $n$ & $\%$ & $\mathrm{n}$ & $\%$ & \\
\hline Alta & 0 & 0 & 4 & 0,04 & 4 \\
\hline Interconsulta & 57 & 0,64 & 82 & 0,78 & 139 \\
\hline Quirúrgica & 24 & 0,27 & 12 & 0,11 & 36 \\
\hline Fallece & 8 & 0,09 & 7 & 0,07 & 15 \\
\hline Total & 89 & 1,00 & 105 & 1,00 & 194 \\
\hline
\end{tabular}

CC: cardiopatías congénitas. No existe diferencia estadísticamente significativa, entre los tipos de resolución de CC con detección pre y post natal en los periodos pre AUGE y AUGE

El fallecimiento se presentó con un porcentaje de $12,3 \%$ en el periodo pre AUGE y se reduce a $10,4 \%$ en periodo AUGE, similar a lo previamente informado en una población de pacientes argentinos (17). De las CC diagnosticadas en el prenatal en periodo pre AUGE, la mortalidad fue de $50 \%$ y con plan AUGE se reduce a $24,1 \%$, por lo tanto en este periodo AUGE los resultados demuestran una mejoría en la sobrevida neonatal secundaria al diagnóstico prenatal.

La información adquirida a través del diagnóstico prenatal es útil para el obstetra en el manejo del embarazo, trabajo de parto y parto y en algunas circunstancias puede mejorar el resultado del embarazo, ya que al estar confirmado el diagnóstico de $\mathrm{CC}$, se puede realizar el traslado de la madre previo al nacimiento, y coordinar la formación de un equipo multidisciplinario con el fin de proporcionar oportunamente al recién nacido el manejo y tratamiento que requiera (21). A este respecto, Chile exhibe las cifras más altas de América Latina en cobertura de control prenatal, partos institucionales y atención de parto por personal capacitado, lo que se refleja en la disminución de la tasa de mortalidad fetal tardía y neonatal precoz (22), que es la base para que las intervenciones pre y postnatales presenten una mayor eficacia y con esto contribuir al cumplimiento de los objetivos sanitarios planteados como metas al 2010. 


\section{CONCLUSIÓN}

Como la mayoría de las CC detectadas en nuestra población, se presentaron en ausencia de factores de riesgo maternos y/o fetales, por lo que cobra vital importancia la implementación del plan AUGE, que produjo un mayor número de diagnósticos de CC, principalmente durante la fase prenatal y confirmado por la introducción de ECGF, todo esto determinó una mayor sobrevida neonatal.

Agradecimientos: Los autores agradecen a Dr. Germán Müllhausen, Jefe de la Unidad de Neonatología del Hospital San José, por permitir el acceso a la información contenida en las bases de datos de pacientes y a los Dres Eduardo Sepúlveda, obstetra del Hospital San José, e Ignacio Hernández, cardiólogo del Hospital Roberto del Río y Gilberto Palominos, Jefe de la Unidad de Cirugía Cardiaca del Hospital Roberto del Río, por su apoyo en el planteamiento y desarrollo del estudio.

\section{BIBLIOGRAFÍA}

1. Ministerio de Salud. Objetivos sanitarios para la década 2000 - 2010. El Vigía. Boletín de Vigilancia en Salud Pública de Chile 2002;5(15):1-12. Hallado en: http://epi.minsal.cl/epi/html/elvigia/vigia15.pdf

2. Departamento de Epidemiología. Departamento de Estadísticas e Información de Salud del Ministerio de Salud. DEIS. Indicadores Básicos de Salud 2002. Hallado en: http://epi.minsal.cl/epi/html/sdesalud/IndSalud2002.pdf

3. Etchegoyen G, Paganini JM. La relación entre los factores socioeconómicos y los programas sanitarios de salud maternoinfantil en 13 provincias argentinas. Rev Panam Salud Publica 2007;21(4):223-30.

4. Jiménez J, Romero MI. Reducing infant mortality in Chile: success in two phases. Health Aff 2007;26(2):45865.

5. Szot J. Mortalidad infantil por malformaciones congénitas: Chile, 1985-2001. Rev Chil Pediatr 2004;75(4):34754.

6. Romero M, Bedregal P, Bastías G. Situación de la salud materno infantil en Chile. Boletín Escuela de Medicina, Pontificia Universidad Católica de Chile 1994;23:10-4.

7. Morera C. Correlación Nacional de la Mortalidad Infantil del Cantón de Grecia. Quinquenio 1996 - 2000: Mortalidad Infantil de un Dígito y Cambio de Estructura Causal en Grecia. Acta Pediatr Costarric 2003, 17(2):40-5.

8. Gobierno de Chile. Ministerio de Salud. Manual de atención personalizada en el proceso reproductivo. Departamento ciclo vital, 2007. Hallado en: http:// www.redsalud.gov.cl/archivos/ciclovital/saludreproductiva.pdf

9. Ministerio de Salud. Objetivos Sanitarios para la Década 2000 - 2010: Evaluación a mitad del período. Hallado en: http://epi.minsal.cl/epi/html/sdesalud/OS/ OS2000_2010.htm\#1

10. Ministerio de Salud de Chile. Guía Perinatal. González R, Gómez R, Castro R (eds.) Santiago, 2003.

11. Ministerio de Salud Pública de Chile. Guía clínica de cardiopatías congénitas operables en menores de 15 años. Hallado en: http://www.minsal.cl/ici/guiasclinicas/CardiopatiaCongenita.pdf

12. Ministerio de Salud. AUGE. Ley $N^{\circ} 19.966$. Hallado en: http://www.bcn.cl/leyes/pdf/actualizado/229834.pdf

13. Martin E, Llanes M, Castillo A, González G, Torres D, Vega $T$. Incidencia de las cardiopatías congénitas en el menor de un año. Villa Clara, 1998 -2002. Rev Costarric Cardiol 2003;5(1):19-24.

14. Hoffman JI. Incidence of congenital heart disease: Postnatal incidence. Pediatr Cardiol 1995;16(3):10313.

15. Stauffer NR, Murphy K. Prenatal diagnosis of congenital heart disease: the beginning. Crit Care Nurs $Q$ 2002;25(3):1-7.

16. Quiroz L, Siebald E, Belmar C, Urcelay G, Carvajal J. El diagnóstico prenatal de cardiopatías congénitas mejora el pronóstico neonatal. Rev. Chil Obstet Ginecol 2006; 71(4): 267- 273

17. Guerchicoff $M$, Marantz $P$, Infante J, Villa A, Gutiérrez A, Montero G, Elias D. Evaluación del impacto del diagnóstico precoz de las cardiopatías congénitas. Arch Argent Pediatr 2004;102(6):445-50.

18. Carvalho S, Mendez M, Cavalli R, Machado J, Duarte G, Berezowski A. Rastreamento e diagnóstico ecocardiográfico das arritmias e cardiopatias congênitas fetais. Rev Bras Ginecol Obstet 2006;28(5):304-9.

19. Bacaltchuk T, Antunes $P$, Zielinsky $P$. Rastreamento pré-natal de anormalidades cardíacas: papel da ultra-sonografia obstétrica de rotina. Rev Bras Ginecol Obstet 2001;23(9):553-8.

20. International Society of Ultrasound in Obstetrics \& Gynecology. Cardiac screening examination of the fetus: guidelines for performing the "basic" and "extended basic" cardiac scan. Ultrasound Obstet Gynecol 2006;27(1):107-13.

21. Cunnif C. Committee on Genetics. Prenatal screening and diagnosis for pediatricians. Pediatrics 2004;114:88994.

22. Donoso E. Mortalidad perinatal en las Américas. Rev Chil Obstet Ginecol 2005,70(5):279-80. 